resident in England and Wales were performed after 20 weeks in 1986 and not one in five, as stated in the report. The rate of complications was also grossly overstated; a major complication occurs after about $0.3 \%$ of abortions at eight weeks, but delaying the abortion until 10 weeks increases the complication rate by $60 \%$ and delaying it until 15 weeks by $300 \%{ }^{1}$.

Everyone wants to reduce the number of late abortions. The conference was called because of the considerable evidence that women often experience difficulty when trying to obtain help from the National Health Service with unwanted pregnancies. This delays abortions and increases the risks at all gestations. In 1981 delay in the National Health Service was the reason why $20 \%$ of women had their abortions after 20 weeks. ${ }^{2}$

The conference discussed means of avoiding delay by better access to pregnancy testing, and examples of efficient National Health Service provision of abortions were described. The proceedings of the conference are to be made available by the Birth Control Trust (price £5.50).

Birth Control Trust,

27-35 Mortimer St,

London W1N 7RJ

1 Grimes DA, Cates W. Complications from legally induced abortion: a review. Obstet Gynecol Surv 1979;34:177-91.

2 Alberman E, Dennis KJ. Late abortions in England and Wales. London: Royal College of Obstetricians and Gynaecologists, 1984.

\section{Tonsillar enlargement and failure to thrive}

SIR,-In their article on tonsillar hypertrophy and failure to thrive (29 August, p 541) Drs $S$ Hodges and $M P$ Wailoo mention some values for oxygen saturation in a child with upper airways obstruction but do not comment further on this aspect of assessment. We would like to illustrate the value of pulse oximetry in such circumstances by a case that we encountered recently.

Our patient, a girl aged 19 months who had been born at 26 weeks' gestation, was growing poorly and was admitted as a day case for investigation. After sedation for removal of a biopsy specimen of the small bowel she showed signs of upper airway obstruction, and examination showed large, although apparently healthy, tonsils. All signs of obstruction abated as the sedation wore off, but she was admitted overnight and monitored by pulse oximetry. Trace 1 shows the serious degree of hypoxia that occurred intermittently during sleep.

This evidence persuaded a previously reluctant ear, nose, and throat surgeon to remove the child's tonsils and adenoids, with the result that trace 2 was obtained 72 hours after operation. Her parents believe that her appetite and general wellbeing
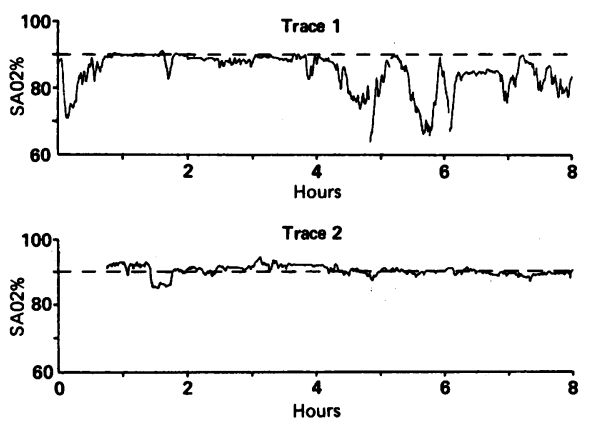

Pulse oximetry traces in patient with upper airway obstruction before and after removal of tonsils and adenoids. began to improve within days of the operation. It is too soon to tell whether she will grow any better.

N J SHAW

I BOWLER

P R F DEAR

St James's University Hospital,

Leeds LS9 7TF

\section{Regional neonatal intensive care: bias and benefit}

SIR,-While I agree with Dr Teyrnon G Powell and Professor Peter O D Pharoah (19 September, $p$ 690) that very low birthweight infants born at the regional intensive care unit probably stand a better chance of survival, care should be taken before these figures are acted on, especially when resources are limited. If regional units are developed at the expense of peripheral ones it is possible that the overall survival will fall.

In a review of all infants born at Leicester General Hospital in the years 1984, 1985, and 1986 ( 9747 deliveries in total) between 24 and 32 weeks and weighing less than $1500 \mathrm{~g}$ it was found that in 22 out of $60(37 \%)$ cases it would have been impossible to transfer them in utero. Therefore if the special care baby unit were closed to provide funding and staff for the neonatal unit at the Leicester Royal Infirmary there would be at least eight cases each year in which the neonatal flying squad would have to be called. An extrauterine transfer of a sick neonate is often an unsatisfactory procedure with an appreciable morbidity.

Other factors that should be taken into account when recommending in utero transfer of small infants are the psychological effect on the mother of moving her from the local hospital and friends and the necessity for junior obstetric staff and midwives in the local hospital to obtain training in the management of premature delivery. Therefore, although in utero transfer sounds attractive, neonatal units should not be developed just so that district administrators may reduce their local hospital costs.

\section{Michael R COHN}

Leicester General Hospital,

Leicester

\section{Health promotion campaigns}

SIR,-Dr Gellise Bagnall and colleagues (12 September, $p$ 660) rightly emphasise the importance of evaluating health campaigns. This is true for campaigns related to the acquired immune deficiency syndrome as well as for all other prevention programmes.

Objective and competent evaluation of this kind of campaign is, however, extremely difficult. Changes in behavioural attitudes are so complex, especially with regard to sex, that a control group is mandatory for measuring the effect of the campaign. As such campaigns are directed at groups individual randomisation is impossible; only randomisation by group may be used. The difficulties of such designs have been pointed out, ${ }^{12}$ but this seems to be the only reasonable means of evaluation.

A controlled study of this kind has been undertaken in France to evaluate the effect of a campaign to prevent the spread of sexually transmitted diseases. Six French departments were randomised, in two groups, three of them to receive the prevention programme and three to act as controls. The incidence of sexually transmitted diseases and the relevant risk factors were measured just before the programme and three months later, using a representative sample of general practitioners in each of the departments ( 261 general practitioners participated).

Initial results indicate that the change in the incidence of sexually transmitted diseases was very similar in all departments irrespective of group (there was a mean decrease from $6.1 \%$ to $4.4 \%$ in the controls and from $5 \cdot 8 \%$ to $4.7 \%$ in the intervention group). More interestingly, a change was noted after the programme in the intervention group in the characteristics of women who consulted about sexually transmitted diseases; the proportion of patients who had had more than one sexual partner in the past three months changed significantly (36\% before, $9 \%$ after), as did the proportion having sex with occasional partners (36\% before, $5 \%$ after) and also the proportion who delayed longer than seven days before consulting $(81 \%$ before, $57 \%$ after). There was no difference between the two groups before the programme. This may be related either to a decrease in exposure to risk factors or to an increased use of protective measures in the exposed subjects-for example, use of condoms. Finally, a survey has indicated that the best way to inform exposed people about sexually transmitted diseases is through comics, posters, and advertising cartoons.

This kind of evaluation is long, difficult, and expensive, but this seems to be the only way to evaluate health promotion campaigns objectively.

\section{N JOB-SPIRA \\ L MEYER E BOUVET-KOSKAS A SPIRA}

INSERM U 292, Santé Publique Epidemiologie Reproduction Humaine, Hôpital de Bicêtre, 94275 Le Kremlin-Bicetre, France

1 Cornfield J. Randomization by group: a formal analysis. Am $\mathcal{J}$ Epidemiol 1978;108:100-2.

2 Bonner A, Birkett N, Buck C. Randomization by cluster. Sample size requirements and analysis. Am J Epidemiol 1981;114: 906-14.

\section{Sexual dysfunction in women with Crohn's} disease

SIR,-Dr Elspeth Guthrie and colleagues reported sexual dysfunction in $83 \%$ of women with the irritable bowel syndrome (5 September, p 577). Thirty per cent of women with inflammatory bowel disease also reported that their sexual activities were affected by their illness. We are currently studying menstrual and menopausal problems among women with Crohn's disease and have noted similar problems among $30 \%$ of our patients.

One hundred and forty five women with Crohn's disease from south Wales ${ }^{1-3}$ and 50 nurses with Crohn's disease ${ }^{4}$ were interviewed about sexual activities, menstruation, and the menopause (table). One hundred and sixty four were sexually active at the time of the study, and 49 reported pain

Dyspareunia among women with Crohn's disease ( $n=$ 195)

\begin{tabular}{lrcc}
\hline & Total & $\begin{array}{c}\text { Sexually } \\
\text { active }\end{array}$ & $\begin{array}{c}\text { Those with } \\
\text { dyspareunia }\end{array}$ \\
\hline $\begin{array}{l}\text { All women } \\
\text { Patients with Crohn's disease: }\end{array} \quad 195$ & 164 & 49 \\
$\quad \begin{array}{l}\text { Menstruating } \\
\text { Amenorrhoeic }\end{array}$ & 83 & 78 & 25 \\
$\begin{array}{l}\text { Nurses with Crohn's disease: } \\
\quad \text { Menstruating }\end{array}$ & 62 & 42 & 10 \\
$\quad$ Amenorrhoeic & 34 & 32 & 11 \\
\hline & 16 & 12 & 3 \\
\hline
\end{tabular}

